\title{
Le dictionnaire comme outil d'enseignement des langues au Gabon
}

\author{
Guy-Modeste Ekwa Ebanéga, Département des Sciences du Langage,
}

Université Omar Bongo, Libreville, Gabon (guymodeste_e@yahoo.fr)

\begin{abstract}
Résumé: Le but de cet article est de montrer dans quelle mesure le dictionnaire peut être un outil d'enseignement des langues gabonaises. Dans un pays, comme le Gabon, caractérisé par une pluralité de langues locales et étrangères, le dictionnaire sera non seulement un ouvrage de référence contenant l'ensemble des mots d'une langue et fournissant pour chacun des données phonétiques, morphologiques, sémantiques, étymologiques etc. Il sera également un outil didactique permettant aux étudiants et aux élèves d'acquérir une compétence nécessaire de leurs langues. Il sera encore un moyen efficace qui va permettre aux usagers de maîtriser leurs langues et dépasser les frontières linguistiques qui existent entre les langues.
\end{abstract}

Mots clés: DICTIONNAIRE, LANGUES GABONAISES, ENSEIGNEMENT, UNITÉS LEXICOGRAPHIQUES, STRUCTURE DU DICTIONNAIRE, PHONÉTIQUE, MORPHOLOGIE, SÉMANTIQUE, VOCABULAIRE, ÉTYMOLOGIE

\begin{abstract}
The Dictionary as Tool for Teaching the Gabonese Languages. The aim of this article is to show to what extent the dictionary can be a tool for teaching the Gabonese languages. In a country like Gabon, characterized by a multiplicity of local and foreign languages, the dictionary will not only be a reference work containing a collection of the words of a language and providing for each its phonetic, morphologic, semantic and etymologic etc. data. It will also be a didactic tool allowing students and pupils to acquire the necessary competence in their languages. It will further be an efficient means which will allow users to master their languages and to overcome the linguistic boundaries existing between the languages.
\end{abstract}

Keywords: DICTIONARY, GABONESE LANGUAGES, TEACHING, LEXICOGRAPHIC UNITS, DICTIONARY STRUCTURE, PHONETICS, MORPHOLOGY, SEMANTICS, VOCABULARY, ETYMOLOGY

\section{Introduction}

Les dictionnaires ont été compilés et utilisés pendant plusieurs siècles (AlKasimi 1977; McArthur 1986). Ils sont nés d'une situation de transfert de connaissance. Le dictionnaire, comme instrument pratique, répond à une pratique de communication au niveau du décodage et il satisfait un type bien défini d'usagers: des étudiants, des élèves et tous ceux qui se trouvent en situation 
d'apprentissage d'une langue étrangère (Gouws 2000). Les dictionnaires sont des livres de mots (au sens premier, «Wörterbuch» en allemand). Ces mots traduisent les pensées d'un groupe ou d'une nation qui se transmettent de personne en personne et de génération en génération. À ce titre, le dictionnaire est un patrimoine linguistique et culturel à léguer aux générations futures.

Trois périodes caractérisent la lexicographie gabonaise (Ekwa Ebanéga et Tomba Moussavou 2008). Pendant la première et la seconde période, les missionnaires sont les premiers à s'intéresser aux langues gabonaises et compileront les dictionnaires du gabonais. Ces dictionnaires sont tous des dictionnaires bilingues à l'usage du public francophone. La troisième période est celle des lexicographes nationaux qui ont reçu une formation de base en lexicographie et en planification lexicographique pour les langues gabonaises.

Le but de cet article est de montrer dans quelle mesure le dictionnaire peut être considéré comme un outil didactique important pour l'éducation des apprenants au Gabon. Notre premier but sera la mise en place d'une politique lexicographique (2), suivi d'une analyse des problèmes lexicographiques liés à l'éducation au Gabon (3), d'une interrogation sur des dictionnaires se prêtant bien au mode d'instruction (4), d'une valorisation du dictionnaire dans le système éducatif gabonais (5), d'une réflexion sur l'établissement des unités lexicographiques du Gabon (6), d'une identification du public visé: les enseignants, étudiants et élèves (7), d'une insistance sur l'importance d'utiliser le dictionnaire comme outil d'enseignement des langues (8) et d'une exposition des aspects de la lexicographie (9).

\section{La mise en place d'une politique lexicographique}

Le Gabon est un pays multilingue. Kwenzi-Mikala (1998: 217) dénombre 64 parlers, parmi lesquels les langues bantoues et pygmées. Sur le plan de l'éducation, la catégorisation retenue distingue la langue officielle, c'est-à-dire le français, et les langues internationales (anglais, espagnol, allemand, portugais, italien et arabe), enseignées aux niveaux secondaire et supérieur. Quelques langues gabonaises telles que le fang, le yipunu, le yinzebi et l'omyéne sont enseignées dans certains établissements secondaires catholiques de Libreville, notamment à l'Institut de l'Immaculée Conception, à Quaben, à Bessieux et à Sainte Marie.

Depuis les États Généraux de l'Éducation Nationale 1983, le gouvernement gabonais s'engage à promouvoir les langues du pays, afin de sauvegarder les cultures gabonaises, ainsi que par l'insertion de ces langues dans l'éducation.

En 1984, le Gabon s'efforce de mettre en place une politique linguistique qui contribuerait au développement de ses langues nationales, aux côtés de la langue française. Au cours des dernières années, la linguistique s'est développée au Gabon, et nous pouvons observer l'apparition des travaux universitaires, qu'il s'agisse des outils pédagogiques et didactiques (livres de lecture ou 
d'écriture, contes, etc.), ou de lexiques pour l'enseignement des langues gabonaises. À l'Université Omar Bongo, des recherches linguistiques se créent pour «penser les langues gabonaises». Nous pouvons citer entre autres le Groupe de Recherche en Langues et Cultures Orales (GRELACO), le Centre de Recherche en Langues et Linguistique (CRELL), le Laboratoire Universitaire de Tradition Orale (LUTO), et le Laboratoire des Sciences de l'Homme et de la Dynamique du Langage (LASCIDYL). Ces structures de recherche sont destinées à sauvegarder et à promouvoir les langues locales pour l'élaboration des projets de recherche.

Un accord de coopération liant le Groupe de Recherches en Langues et Cultures Orales (GRELACO) de l'Université Omar Bongo et le Bureau du Woordeboek van die Afrikaanse Taal (WAT) fut signé le 6 décembre 1999. Également en 1999, deux étudiants gabonais sont venus au Bureau du WAT pour une formation continue et pour leur étude doctorale en lexicographie à l'Université de Stellenbosch (République d'Afrique du Sud) sous la direction du professeur R.H. Gouws. En 2000, trois autres étudiants sont venus en Afrique du Sud et en 2001, ils ont été suivis par cinq autres. Le Bureau du WAT leur a donné une formation pilote en lexicographie générale, en lexicographie informatique et en planification des projets lexicographiques. Cinq étudiants ont soutenu leur thèse de doctorat en lexicographie.

Trois ans plus tard s'est tenue à Libreville du 18 au 23 juillet 2004, la Neuvième Conférence Internationale de l'Association Africaine pour la Lexicographie (AFRILEX). Le sujet de cette conférence était la problématique du dictionnaire, le développement des langues et l'organisation des centres lexicographiques. Son objet était la recherche des zones de convergences entre le dictionnaire et la langue, afin de proposer une stratégie dynamique pour le développement de la lexicographie gabonaise. Le dictionnaire est un moyen qui rend accessibles les connaissances du monde contemporain aux élèves et aux étudiants qui sont appelés à le consulter pour la pluralité des langues et des cultures du Gabon.

Depuis 2004, la lexicographie est enseignée au Département des Sciences du Langage à l'Université Omar Bongo. On y dénombre, en ce moment, quatre lexicographes-enseignants. Pour la période 2006-2008, le CENAREST (Centre National pour la Recherche Scientifique et Technologique) a, à son tour, recruté deux lexicographes-chercheurs.

\section{Les problèmes lexicographiques liés à l'éducation au Gabon}

Il semble toutefois que les dictionnaires existant en langues gabonaises sont très peu utilisés au Gabon. Ils ne font pas partie des ouvrages courants à consulter, et ils sont rangés dans la catégorie des ouvrages occasionnels. Les dictionnaires en langues gabonaises ne se trouvent pas dans les librairies, ni sur les rayons des bibliothèques générales, ni dans les établissements scolaires et universitaires. 
L'observation de McKean à propos des jeunes enseignants d'anglais est aussi valable pour les jeunes enseignants gabonais pour qui leur cours n'implique aucune information sur les dictionnaires et rien sur l'instruction des connaissances dictionnairiques. Il faut noter que bon nombre d'enseignements sont dispensés en langues étrangères (français, anglais, allemand, espagnol, etc.) et les étudiants sont familiers avec ces langues. Cela n'est pas le cas des langues $\mathrm{du}$ Gabon où nous notons une absence non seulement de l'enseignement des langues nationales mais aussi d'un enseignement des connaissances phonétiques, phonologiques, grammaticales, morphologiques, syntaxiques, sémantiques et pragmatiques de ces langues.

Le gouvernement gabonais ne fournit pas de dictionnaires ou autres ouvrages lexicographiques (qui existent en langues gabonaises) aux écoles; il ne subventionne même pas l'acquisition de ces matériaux pour stimuler les études appropriées. La question que nous pouvons poser consiste à savoir comment les connaissances dictionnairiques peuvent être appliquées et encouragées dans un environnement où elles ne font pas partie du programme d'étude offert dans les établissements, les universités et les cours offerts dans les écoles?

Certaines écoles n'ont pas de bibliothèques et n'encouragent pas la consultation des dictionnaires pour aider l'usager à acquérir les techniques et connaissances dictionnairiques. Ou sinon, la question existe combien de dictionnaires et de types de dictionnaires devraient être maintenus dans les bibliothèques envisagées?

Bon nombre d'enseignants de langues n'ont pas de dictionnaires en classe. Les dictionnaires qu'on trouve dans les salles de classes sont, le cas échéant, destinés à l'usage personnel de l'enseignant, et non pas pour encourager les apprenants à les utiliser.

Certains parents préfèrent acheter des manuels bibliques transcrits en langues (catéchismes, cantiques, évangiles, etc.), ou d'outils pédagogiques et didactiques (livres de lecture ou d'écriture, syllabaires, etc.) qui se trouvent disponibles sur le marché. Cela n'est pas le cas des dictionnaires en langues gabonaises qui deviennent de plus en plus introuvables. Les dictionnaires anglais, français, espagnols et portugais font partie des ouvrages didactiques, or pas les dictionnaires en langues gabonaises.

Les enquêtes menées par Ekwa Ebanéga et Tomba Moussavou (2008) ont montré qu'à la question quel(s) type(s) de dictionnaire(s) existent en langues gabonaises, $36 \%$ des étudiants ont répondu en faveur du dictionnaire général, $10 \%$ pour le dictionnaire spécialisé, $46 \%$ pour le dictionnaire bilingue, et $46 \%$ pour l'encyclopédie. Ekwa Ebanéga et Tomba Moussavou (2008) ont montré que les différentes réponses données par les étudiants sont la preuve qu'ils ne savent pas quel type de dictionnaire existe en langues gabonaises. Les dictionnaires existants en langues gabonaises ne sont pas connus par le public, et particulièrement par les étudiants et les élèves. Ekwa Ebanéga et Tomba Moussavou (2008) ont suggéré qu'un cours sur l'histoire de la lexicographie gabonaise, dont le but est de promouvoir les dictionnaires existants, devrait être enseigné 
dans les écoles, les collèges, les lycées et les universités.

Les recherches faites par Kipfer (1987: 45) ont montré que les étudiants sont réticents à consulter les dictionnaires pour la simple raison qu'ils n'ont pas assez de compétence pour trouver les réponses aux innombrables problèmes auxquels leur langue fait face. Selon lui, la plupart des étudiants qui n'ont pas reçu des enseignements sur le dictionnaire consulte le dictionnaire pour trouver l'orthographe et la signification/l'équivalent du mot. Les informations telles que les exemples et les collocations sont rarement utilisées. L'observation de Kipfer (1987) est similaire aux résultats trouvés par Ekwa Ebanéga et Tomba Moussavou (2008) où 74\% des étudiants gabonais consultent les dictionnaires pour trouver la signification/l'équivalent du mot et $58 \%$ consultent le dictionnaire pour trouver l'orthographe du mot.

Les linguistes et les lexicographes ne sont pas nombreux au Gabon. On y dénombre environ vingt linguistes et dix lexicographes.

Certaines personnes pensent qu'on n'a pas besoin de faire de longues études pour produire des dictionnaires. Il suffit simplement de recueillir les mots.

Bon nombre de personnes pensent que la promotion et le développement des langues sont l'affaire des linguistes seuls, ignorant qu'elles sont les garants de son usage et les acteurs de son évolution.

Certaines personnes pensent que le lexicographe n'est pas fait pour enseigner. Cependant, elles ignorent que, pour être lexicographe, il faut avoir suivi, au préalable, une formation lexicographique. Les lexicographes gabonais ont reçu cette formation qui donne accès au doctorat (cf. Gouws 2001: 98). L'auteur continue en affirmant que l'importance et la valeur académique ne doivent jamais être sous-estimées.

\section{Les dictionnaires et les langues nationales}

La question du pour ou contre du dictionnaire comme outil d'enseignement dans le système éducatif gabonais a été longuement discutée par Ekwa Ebanéga (2007), Tomba Moussavou (2007), Nyangone Assam (2001) et Mabika Mbokou (2001), ces auteurs soulignant l'importance du dictionnaire dans l'enseignement des langues gabonaises. Ceci nous amène à nous interroger sur les langues qui seront utilisées comme mode d'instruction, c'est-à-dire les langues d'enseignement et d'apprentissage dans le système éducatif gabonais. Moyo (2002: 151) envisage l'activité de l'enseignement des langues de deux manières:

(a) Le mode d'instruction, c'est-à-dire la langue dans laquelle communiquent l'apprenant et l'enseignant, est la langue par laquelle l'enseignant transmet le savoir à l'apprenant.

(b) La matière d'apprentissage, c'est-à-dire la langue introduite dans le système éducatif pour acquisition par les apprenants, la langue est ici le savoir à transmettre. 
L'observation faite par Moyo (2002) relativement à l'enseignement et à l'apprentissage des langues, nous amène à voir le statut des langues gabonaises. Kwenzi-Mikala (1998) dénombre 64 parlers, parmi lesquels les langues bantoues et pygmées. Le français jouit d'un statut particulier au Gabon, c'est la seule langue officielle, l'unique mode d'instruction du système éducatif national, des affaires économiques et commerciales, de l'administration civile et militaire (Nyangone Assam et Mavoungou 2000, Ndinga-Koumba-Binza 2005). Sur le plan de l'éducation, la catégorisation retenue distingue la langue officielle, c'est-à-dire le français, et les langues internationales (anglais, espagnol, allemand, portugais, italien et arabe), enseignées aux niveaux secondaire et supérieur. Quelques langues gabonaises telles que le fang, le yipunu, l'omyéné et le yinzebi sont enseignées dans certains établissements secondaires catholiques de Libreville. Nous pouvons citer entre autres l'Institut Immaculée Conception, à Quaben, à Bessieux et à Sainte Marie.

Ainsi, pour le cas du Gabon, nous pensons que le dictionnaire comme instrument d'enseignement des langues gabonaises doit se faire à partir du primaire voire dès la maternelle. Une enquête menée par Ekwa Ebanéga et Tomba Moussavou (2008) est présentée ici pour confirmer ce fait.

Par ailleurs nous pensons également qu'un outil d'enseignement comme le dictionnaire doit aller de pair avec l'intégration des langues gabonaises dans le système éducatif. Une enquête menée par Bokoko (2004) auprès des acteurs des langues nationales (professeurs et membres de l'administration scolaire) est une preuve manifeste pour confirmer cette assertion. Le but de l'enquête était de vérifier l'exactitude des informations sur l'intégration des langues nationales. Selon le vœu exprimé par la majorité des informateurs, les langues nationales devraient être enseignées à partir du primaire aux seules fins d'éviter une rupture brutale avec le milieu familial.

Ainsi, pour le cas du Gabon et prenant en compte les enquêtes menées par Ekwa Ebanéga et Tomba Moussavou (2008) et Bokoko (2004), un outil d'enseignement comme le dictionnaire doit aller de pair avec l'intégration des langues gabonaises. Leur intégration devrait permettre de

- préserver les racines et les cultures gabonaises,

- faciliter l'apprentissage d'autres langues,

- enrichir et améliorer l'expression,

- renforcer l'unité et l'identité nationales, et

_ pallier à l'absence de communication en langues dans les foyers.

Pour permettre aux langues gabonaises d'être enseignées par le biais des dictionnaires, il faut que l'État gabonais définisse une politique claire en matière de la promotion des langues nationales. Pour ce faire, il faudrait répondre aux questions suivantes: 
- Veut on introduire le dictionnaire comme moyen d'enseignement des langues au Gabon?

- Veut on utiliser le dictionnaire comme marche-pied pour faciliter l'apprentissage de nos langues?

En d'autres termes, il faut que les responsables gabonais répondent à la question suivante: dans quel but faut-il créer des dictionnaires de langues gabonaises? Des réponses objectives à ces questions permettront d'accorder une place de choix au dictionnaire. Cependant le bref aperçu sur la place du dictionnaire dans l'enseignement des langues montre que l'État gabonais n'a pas encore trouvé de réponse à ces interrogations. Une fois que les responsables du système éducatif auront trouvé une réponse à ces questions, l'État devrait s'appliquer davantage aux innovations susceptibles d'entraîner un renforcement de la politique linguistique dans le pays.

\section{Valoriser le dictionnaire dans le système éducatif gabonais}

La valorisation du dictionnaire dans l'enseignement des langues gabonaises n'est possible qu'avec l'organisation des conférences, des ateliers et des séminaires dans le but d'instruire les enseignants et les étudiants sur le rôle, la structure et le contenu des dictionnaires. En 1981, par exemple, le linguiste et lexicographe tchèque Ladislav Zgusta a mené un atelier de table ronde sur les dictionnaires bilingues à l'Université d'Illinois. Plus récemment, Kenneth Hill du Bureau de la Recherche Appliquée en Anthropologie, l'Université d'Arizona, a enseigné un cours de deux semaines sur la réalisation du dictionnaire à 20 étudiants dans le programme de linguistique à l'Université de Sonora. À cet effet, il a partagé avec les étudiants ce qu'il avait appris en faisant un dictionnaire hopi aux seules fins de les guider dans la réalisation des dictionnaires. Gates (1997) souligne que depuis 1979, plusieurs ateliers se sont organisés dans au moins sept pays. En 1978, Reinhard Hartmann a organisé un séminaire sur la lexicographie à l'Université d'Exeter financée par l'Association Britannique de Linguistique Appliquée. En mars 1989, le Centre de Recherche du Dictionnaire à Exeter a tenu un atelier sur la lexicographie africaine. En Afrique, il se tient chaque année une conférence sur la lexicographie africaine organisée par AFRILEX (l'Association Africaine pour la Lexicographie), et certains ateliers sont tenus en collaboration avec AFRILEX.

Les initiatives, ci-dessus, mentionnées au Gabon, auront essentiellement pour objectif la valorisation du dictionnaire dans le système éducatif. En termes plus concrets, la tenue des conférences, des séminaires et des ateliers au Gabon permettront aux enseignants et étudiants de se familiariser avec les connaissances dictionnairiques. Les lexicographes gabonais pourraient, par exemple, organiser au Gabon un séminaire dont l'objet est "le dictionnaire comme instrument d'enseignement des langues". Ce séminaire aurait comme slogan "Donne moi 
ton dictionnaire, j'évaluerai ta langue". ${ }^{1}$ On pourrait expliquer ce slogan en disant qu'on ne peut pas espérer une éducation efficiente et développée sans considérer une langue. La connaissance linguistique se trouve dans le dictionnaire qui contient des données linguistiques telles que la phonétique, la phonologie, la morphologie, la syntaxe, la sémantique, d'où la nécessité de promouvoir le dictionnaire. Nul n'ignore que toute connaissance transmise dans la langue maternelle est mieux comprise que si elle était transmise dans une langue étrangère. Les dernières recherches faites par Joubier (2008) ${ }^{2}$ le prouvent. On apprend mieux dans sa langue maternelle. Les enfants dont l'éducation a commencé dans leur langue maternelle prennent un meilleur départ, et réussissent mieux par la suite, que ceux dont la scolarité a débuté dans une langue autre que la leur.

\section{L'établissement des unités lexicographiques}

La question de l'établissement des unités lexicographiques est un facteur déterminant si l'on veut que le dictionnaire soit un instrument d'enseignement des langues gabonaises. Une unité lexicographique est une structure de recherche dont l'objectif principal est la production pratique des dictionnaires. Dans le cadre de la politique linguistique des langues africaines, le projet de type PanSALB (Pan South African Language Board) ${ }^{3}$ pour les langues sud-africaines a été établi par le gouvernement de l'Afrique du Sud en 1995. Pour le cas du Gabon, un projet de type PanSALB aurait pour résultat la préservation de la diversité linguistique et l'établissement des onze unités lexicographiques telles que les unités françaises et mazuna, myene, mekana-menaa, mekona-mangote, membe, merye, metye, membere, makena, baka, et selon les dix groupes de langues ${ }^{4}$ préalablement cités. Celles-ci serviraient d'interface pour la collaboration entre les lexicographes et les linguistes. En établissant les unités lexicographiques au Gabon, ceci aurait comme résultat la production des dictionnaires et la préservation de la diversité linguistique sous toutes ses formes variées. L'établissement des unités lexicographiques devrait aller de pair avec la politique linguistique du pays. Les actions en faveur de la préservation de la diversité linguistique devraient être prises par le gouvernement. Nous pourrions citer entre autres l'enseignement des langues gabonaises dans les écoles, lycées, et universités gabonais; l'utilisation des langues gabonaises dans les médias (radios, net et presse) et la reconnaissance constitutionnelle et légale aux langues gabonaises. La prise en compte de ces actions par le gouvenement gabonais serait plus que vitale si nous voulons que l'établissement des unités lexicographique soit effectif.

La création d'une unité lexicographique est de rigueur compte tenu des besoins lexicographiques des langues gabonaises (Nyangone Assam et Mavoungou 2000: 252; Nyangone Assam 2001: 188). Cette structure est en train de se mettre en place et sera fonctionnelle dans le cadre du Groupe de Recherche en Langues et Cultures Orales (GRELACO) au sein de l'Université Omar Bongo 
(Emejulu et Nzang-Bié 1999). Le GRELACO œuvre dans le sens de la création des unités lexicographiques avec le concours des communautés linguistiques. Pour satisfaire les besoins en matière de dictionnaires et produits dérivés, supports indispensables pour le développement et la standardisation des langues, il est d'une nécessité absolue de créer des unités lexicographiques à travers l'ensemble du territoire gabonais, et ce en fonction des besoins spécifiques des langues et des communautés linguistiques telles que les communautés françaises et mazuna, myene, mekana-menaa, mekona-mangote, membe, merye, metye, membere, makena, baka, et suivant les dix groupes de langues. Selon Emejulu (2001: 50), il est plus pratique et économique d'établir les unités lexicographiques dans les localités où se trouvent les unités-langues répertoriées. Les unités lexicographiques seront installées auprès des académies provinciales dans d'autres provinces que la province du Haut-Ogooué dont l'unité lexicographique sera installée au sein de l'Université des Sciences et Techniques de Masuku à Franceville. L'unité lexicographique du français sera établie à Libreville, la capitale du Gabon. Pour le cas de l'unité lexicographique du français, elle prendra en compte la variété du français au Gabon (Mavoungou 2002).

Toutes ces unités lexicographiques seront animées par des agents lexicographiques formés par le GRELACO. La première tâche des unités lexicographiques sera l'identification et la planification des projets lexicographiques à court, moyen et long termes. La deuxième et principale tâche est d'assumer la responsabilité du développement des corpus de différentes langues.

\section{Le public visé: enseignants, étudiants et élèves}

Le dictionnaire comme outil d'enseignement des langues est destiné aux personnels enseignants et aux étudiants qui auront reçu une formation de lexicographes. Ils auront des connaissances spécifiques sur des langues et la compétence de leurs langues maternelles respectives, ils auront besoin également des qualifications générales, d'expertises lexicographiques, d'expertises métalexicographiques, et, en d'autres termes, d'une formation appropriée (Gouws 2001: 99). Les enseignants doivent, à leur tour, transmettre leur savoir aux élèves et aux étudiants. Dolezal et McCreary (1999: IX) affirment: "Puisque nous observons le développement futur de cette discipline, les soucis mutuels du professeur, du lexicographe et de l'apprenant du dictionnaire pourraient être le centre de la lexicographie pédagogique." Les enseignants, comparés aux autres chercheurs ou lexicographes, sont les mieux placés pour évaluer les besoins des étudiants et élèves. Si les enseignants reçoivent une formation de base en lexicographie et, en retour, les transmettent aux étudiants et élèves, la culture du dictionnaire pourrait se développer.

Pour le cas du Gabon, je propose que les enseignants reçoivent une formation de base en lexicographie. Ils doivent également avoir des connaissances particulières sur les langues gabonaises. Cette formation servira d'interface pour la collaboration entre les linguistes et les lexicographes qui sont appelés à 
travailler ensemble dans le but de préserver la riche variété des langues gabonaises (Ekwa Ebanéga et Tomba Moussavou 2006: 243). Les étudiants et élèves profiteront du savoir des enseignants et pourront enrichir leurs connaissances.

\section{L'importance d'utiliser le dictionnaire comme outil d'enseignement des langues}

Pour bien comprendre l'importance d'utiliser le dictionnaire comme outil d'enseignement des langues, les enseignants et étudiants sauront que le dictionnaire est le récipient du savoir. L'enquête menée par Ekwa Ebanéga et Tomba Moussavou (2008) auprès des étudiants gabonais à l'Université de Stellenbosch et l'Université de Technologie de la Cape Peninsula confirme que $89 \%$ des étudiants pensent qu'il est important de recevoir des connaissances linguistiques par le biais de l'outil qu'est le dictionnaire. La culture du dictionnaire pourrait se développer au Gabon, particulièrement parmi les élèves et étudiants en visant d'abord les enseignants. Cependant, les étudiants ne peuvent pas acquérir des connaissances linguistiques par eux-mêmes sans l'aide des professeurs. Le centre d'intérêt serait dédié à la formation des professeurs et enseignants. Selon Hartmann (2001: 26), les enseignants ont rarement reçu la formation requise pour juger les avantages et les compétences dictionnairiques appropriées pour garantir les meilleures conditions de consultation réussie. Ils ne sont que rarement en contact avec des lexicographes ou des chercheurs de dictionnaire.

Le manque de prise en compte du dictionnaire comme outil d'enseignement des langues dans le monde en général et au Gabon en particulier est commun dans la plupart des langues du monde. Landau (2001: 26) note cela: "C'est une pitié, parce que l'habitude de consulter un dictionnaire est formée tôt dans la vie, et si les qualifications pour l'utiliser sont négligées, les étudiants ne peuvent jamais être les usagers confortables du dictionnaire". Cette observation vaut pour des usagers gabonais en général et les usagers en particulier. L'utilisation du dictionnaire doit faire partie intégrante de la formation de l'enseignant. La culture du dictionnaire doit être instaurée en passant par l'enseignant. C'est l'une des raisons pour laquelle Wiegand (1984) affirme que ce n'est pas l'autorité du dictionnaire qui est mise en doute, mais qu'il faut instaurer une culture du dictionnaire.

Par ailleurs, il faut dire que le dictionnaire est né d'une situation d'apprentissage. L'importance d'utiliser le dictionnaire comme outil d'enseignement de langues reposerait sur le fait que le dictionnaire répond à un besoin de communication aux niveaux du décodage (réception) et de l'encodage (production). Au niveau de la réception, lorsque les étudiants et les élèves ne connaissent pas le sens d'un mot, le dictionnaire fournit des données suivantes: la définition, le synonyme, l'antonyme, etc. Au niveau de la production, lorsqu'ils n'arrivent pas à produire un mot, le dictionnaire leur fournira des données comme la prononciation, la morphologie, etc. 
Dans le cas de l'enseignement des langues gabonaises, le dictionnaire devrait, tout d'abord, aider les étudiants et les élèves à acquérir la compétence nécessaire dans une langue. Ensuite, ils feront appel au dictionnaire, lorsque se poseront des problèmes de communication aux niveaux de la réception et de la production.

Dès que les connaissances linguistiques seront acquises chez les enseignants, on pourrait aussi bien compléter leur formation en leur enseignant plusieurs aspects de la lexicographie. Ces aspects sont les suivants:

\section{Les aspects de la lexicographie}

\subsection{La métalexicographie}

Les enseignants auront une formation de la théorie et la pratique des dictionnaires. Cette formation reposera sur les principes qui gouvernent les dictionnaires existants conduisant à la formulation des hypothèses orientées vers la production de bons dictionnaires. Les aspects suivants de la métalexicographie seront pris en compte au cours de cette formation:

- la recherche sur l'utilisation des dictionnaires,

- l'histoire de la lexicographie,

- la critique des dictionnaires, et

- la théorie de la lexicographie générale.

Pour ce qui est de la théorie de la lexicographie générale, les aspects suivants seront également pris en compte (Hausmann 1986 et Wiegand 1984):

- la théorie textuelle des textes lexicographiques,

- les types de dictionnaires,

- la collection et l'assemblage des données, et

- les buts des dictionnaires.

Selon Gouws (2001: 99), la formation en lexicographie et métalexicographie doit inclure des matières suivantes:

- différents types de dictionnaires,

- structure des dictionnaires,

- compilation d'un livre d'instruction pour le dictionnaire donné,

- différents types de lemmes,

- choix de lemmes pour un dictionnaire spécifique, 
- différentes manières pour ordonner les lemmes,

- différents types de catégories de données dans un dictionnaire,

- comment écrire une définition du dictionnaire,

- comment utiliser les exemples d'illustration dans un dictionnaire,

- choix de l'équivalent correct pour la traduction, et

- comment indiquer la prononciation correcte.

Les étudiants et les élèves qui sont des lexicographes en devenir auront une connaissance générale sur les aspects de la métalexicographie. Lorsque les problèmes d'ordre théorique se poseront, les enseignants formés pourront richement illustrer ceux-ci avec des exemples pris dans la pratique lexicographique. Les programmes à enseigner seront élaborés par des lexicographes et des enseignants en fonction des cycles et des objectifs voulus.

\subsection{L'histoire de la lexicographie gabonaise}

Il importe que les enseignants aient une connaissance satisfaisante sur l'origine et les débuts de la lexicographie en langues gabonaises. Toutefois, ils inculqueront aux étudiants que les premiers dictionnaires en langues gabonaises ont été compilés par les missionnaires vers les années 1800. Mihindou 2001 en rend compte au cours d'une enquête sur le nombre de dictionnaires qui existent en langues gabonaises. L'auteur dénombre une quinzaine de dictionnaires disponibles. Pour de plus amples précisions, voir Mihindou (2001: 11-12). Ces ouvrages constituent une excellente source d'informations sur l'histoire de la lexicographie au Gabon. Ils pourraient servir de départ à la constitution d'une base de données en vue de la compilation des dictionnaires dans les langues gabonaises. Les enseignants montreront aux étudiants les avantages et les limites des dictionnaires existants. Il importe de garder en mémoire que les premiers dictionnaires compilés en langues gabonaises avaient pour rôle majeur de servir de support pédagogique à la lecture des langues gabonaises. Ils n'y avait pas, comme aujourd'hui, un mouvement conjugué de la pratique et la théorie des dictionnaires, cf. Mihindou (2001).

Les données présentées dans les pré-textes du dictionnaire sont des données importantes pour l'acquisition de l'histoire d'une langue. Prenons le cas des pré-textes du Dictionnaire fang-français et français-fang produit par Galley (1964), qui présente un texte composé de données sur l'histoire du peuple fang, l'histoire de leur langue et les idées qui ont guidé les recherches précédentes. Ces données sont des données d'arrière-plan (Ekwa Ebanéga 2007: 171). Les étudiants et les élèves pourront tirer là d'importantes informations concernant leur origine et leur passé. L'enseignant expliquera aux élèves et aux étudiants qu'en dehors de la nomenclature (la deuxième composante du dictionnaire) un 
traitement additionnel des données du dictionnaire est aussi offert dans un autre texte du dictionnaire. Ils pourront alors accéder à ces données de plusieurs façons.

\subsection{Les groupes cibles}

La typologie des dictionnaires doit reposer sur l'identification des besoins des utilisateurs cibles, cf. Gouws (2001: 111), Householder (1962: 279). Sur ce point il est important de préciser que le choix d'un dictionnaire doit préalablement faire l'objet d'une analyse détaillée des besoins des utilisateurs ou groupes cibles. La tâche de l'enseignant est, en premier lieu, d'identifier les différents usagers (étudiants ou écoliers) afin de pouvoir arriver à déterminer les besoins qui leur sont propres. Le groupe d'étudiants spécialistes nécessite des dictionnaires spécialisés en rapport avec leur champ d'étude. Dans le domaine de la science, par exemple, il y a des dictionnaires de médecine, de biologie, de physique; dans le domaine des humanités, il y a des dictionnaires de philosophie, d'anthropologie, de sociologie, de littérature. Pour ce qui est de la structuration linguistique du Gabon en général et du fang en particulier, Nzang-Bié (2004) affirme qu'il est nécessaire d'identifier au préalable l'utilisateur cible si l'on veut déterminer la fonction que l'on veut assigner au dictionnaire, car de lui dépend tout le processus du dictionnaire. Dans le cadre du fang, l'auteur distingue trois catégories d'utilisateurs cibles:

- catégorie 1: l'utilisateur cible ne sachant ni lire, ni écrire encore moins parler sa langue;

- catégorie 2: l'utilisateur cible ne sachant ni lire, ni écrire, mais sachant parler sa langue; et

- catégorie 3: l'utilisateur cible sachant lire, écrire et parler sa langue.

Il est à noter que les catégories 1 et 2 identifiées par Nzang-Bié (2004) sont uniquement pour le débutant. Alors que pour la catégorie 3, on peut distinguer le débutant, l'intermédiaire et l'avancé. Le but de l'enseignant est de pouvoir guider les usagers composés d'écoliers, d'étudiants, d'enfants et d'adultes sur le type de dictionnaire à choisir aux seules fins de satisfaire leurs besoins.

Il faut noter qu'à chaque type d'usager correspond un niveau d'apprentissage bien précis.

\subsection{Les types de dictionnaires}

Dès que l'enseignant connaîtra les besoins spécifiques des usagers, il devra être en mesure de les guider quant au choix des dictionnaires capables de répondre à leurs besoins. Mais avant de discuter du type du dictionnaire proprement dit, il faut savoir que le choix du dictionnaire doit tenir compte du contexte socio- 
culturel du pays. Pour le cas du Gabon, l'enseignant orientera les usagers qui veulent apprendre dans la langue étrangère à choisir un dictionnaire bilingue qui comprendrait des informations telles que les équivalences. Il doit guider les usagers qui veulent communiquer dans leur langue à opter pour un dictionnaire monolingue qui apporterait une description assez explicite de la langue. Ce dictionnaire comprendrait des informations telles les définitions, la prononciation, etc. Il résulte de ce qui précède que le type de dictionnaire qui répondrait mieux aux attentes des usagers est le dictionnaire hybride qui englobe les caractéristiques d'un dictionnaire monolingue et celles d'un dictionnaire bilingue. Ce modèle sera intégral et comprendra non seulement le plus grand nombre d'informations linguistiques (définitions, équivalences, etc.), mais il apportera aussi des informations extralinguistiques. Lorsque, par exemple, se posent les problèmes sémantiques entre les élèves de cultures différentes, les illustrations peuvent couvrir le sens du mot.

Brewer (2006) affirme que l'un des problèmes auxquels les lexicographes et la lexicographie font face en ce moment est de savoir comment éduquer les usagers à comprendre les buts de chaque type de dictionnaire et quelles sont les différences entre les types de dictionnaires. Pour ce qui est du Gabon, l'enseignant doit connaître au préalable le but véritable de tout type de dictionnaire et les différences entre les types de dictionnaires. Cette connaissance est nécessaire car elle va permettre à l'enseignant non seulement de guider les étudiants à utiliser et à consulter les dictionnaires convenablement, mais aussi pour conseiller aux écoles comment acheter les dictionnaires qui sont capables de répondre à leurs besoins. La connaissance de la typologie du dictionnaire aidera à élaborer la structure du dictionnaire (Hadebe 2004).

Il a été dit plus haut que le but du dictionnaire est de répondre à un besoin de communication. Pour le cas du Gabon, les enseignants orienteront les étudiants à consulter un dictionnaire qui satisfera leurs besoins. C'est ainsi que lorsqu'ils se trouveront, par exemple, en situation d'apprentissage d'une langue étrangère et seront confrontés par des problèmes de communication au niveau de la production, les enseignants pourront guider les étudiants et les élèves à utiliser un dictionnaire monolingue. Lorsqu'ils seront confrontés par le problème de communication au niveau de la réception, les enseignants les aideront à utiliser un dictionnaire bilingue.

\subsection{La structure du dictionnaire}

Les enseignants apprendront aux étudiants les différentes structures du dictionnaire (la macrostructure, la microstructure, la structure d'accès, la structure d'adressage etc.) et l'importance de chaque structure du dictionnaire. Les étudiants doivent être en mesure de savoir comment utiliser les textes externes du dictionnaire (les pré-textes et posttextes), de pouvoir interpréter les symboles des abréviations, et des explications qui se trouvent dans ces textes externes du dictionnaire. L'apprentissage des structures du dictionnaire est nécessaire pour 
les étudiants car tous les textes du dictionnaire ne sont pas intégrés dans la nomenclature. Les enseignants inculqueront aux étudiants ces textes qui ne sont pas intégrés au dictionnaire pour compléter leur information, cf. Gouws (2001: 123).

\subsection{La morphologie}

Puisque les dictionnaires, comprennent la formation des mots et leur morphologie, l'enseignant prendra soin d'insister sur les différents éléments qui composent le lemme. L'identification de ces éléments crée un problème important sur la façon de déterminer ce qui devrait être identifié comme lemme et ce qui ne devrait pas. À cet effet, Bwenge (1989: 5) notait:

Le problème central est en particulier la méthode d'arranger les articles nominaux et verbaux de la langue, émanant de la structure morphologique complexe commune aux langues bantoues, d'un système de classification morphologique classant des noms par catégorie au moyen des affixes dérivationnels.

Compte tenu de ce qui précède, Ekwa Ebanéga (2007) a signalé que la présentation des données morphologiques (indication de ce qui sont le préfixe, la tige et le nombre de classe) pour chaque signe de lemme peut être compliquée pour les élèves débutants. Par ailleurs, la décision concernant ce qu'il faut inclure ou exclure concernant des données sur la partie du discours doit être faite selon les besoins et les qualifications de référence des utilisateurs.

Dans les posttextes du Dictionnaire fang-français et français-fang, l'auteur présente un texte nommé la minigrammaire dans laquelle les élèves et étudiants pourraient se familiariser avec certains aspects de la structure de la langue fang, à savoir les classificateurs, les préfixes, etc. L'enseignant fournirait aux élèves et aux étudiants des conseils pratiques et des informations sur l'emploi des éléments morphologiques.

\subsection{La phonétique}

La phonétique est une donnée nécessaire du dictionnaire. Le dictionnaire est non seulement un répertoire de mots, mais également un registre des sons d'une langue. Pour connaître une langue, nous devons apprendre les sons de cette langue. En 1954, Malmberg (cité par Berri et Pagel 2005: 1) soulignait déjà l'importance de la phonétique dans l'apprentissage des langues:

L'enseignement des langues étrangères est aussi un domaine où la phonétique a une très grande importance pratique. Celui qui veut apprendre à bien prononcer une langue étrangère, devra acquérir d'abord la maîtrise d'un grand nombre d'habitudes articulatoires nouvelles (une base articulatoire). Il ne faut pas croire qu'il s'agisse seulement d'apprendre quelques sons nouveaux et, pour le reste, utiliser les sons déjà connus. C'est un système d'habitudes articulatoires, y com- 
pris l'intonation et l'emploi des accents expiratoires, qui sera remplacé par quelque chose de nouveau.

Les enseignants montreront aux étudiants les différents alphabets existant en langues gabonaises comme l'Alphabet des Langues du Gabon (ASG) et l'Orthographe des Langues Gabonaises (OLG). La connaissance des alphabets existant en langues gabonaises permettra aux étudiants de connaître les phonèmes qui existent dans les langues gabonaises. Pour ce qui est de la langue fang (ntumu), les étudiants sauront qu'on distingue neuf voyelles (i, e, $\varepsilon, y, \partial, a, u, o$, o) et plusieurs consonnes ( $\mathrm{p}, \mathrm{b}, \mathrm{m}, \mathrm{n}, \mathrm{t}, \mathrm{d}, \mathrm{k}, \mathrm{g}, \mathrm{f}, \mathrm{v}, \mathrm{s}, \mathrm{z}, \mathrm{f}, \mathrm{l}, \mathrm{r}$, etc.). La connaissance des variantes allophoniques de certains phonèmes de la langue fang est aussi nécessaire. Par exemple, la consonne /b/ est réalisée [p] en fin de mot ou de phrase et /b/ ailleurs. Exemple:

$$
\begin{aligned}
& \text { /ekob/ } \rightarrow \text { [éks̀p] "la peau" } \\
& \text { /bəfam/ } \rightarrow \text { [bə́fam] "les hommes" }
\end{aligned}
$$

Les étudiants devraient aussi se familiariser avec les types de tons qui existent en langues gabonaises. La connaissance de la typologie des tons aidera les étudiants à bien prononcer les mots de la langue. Dans la majorité des langues bantoues en général et les langues gabonaises en particulier, les tons jouent un rôle distinctif dans la mesure où ils permettent de distinguer deux mots. Exemples:

$$
\begin{array}{ll}
\text { pové: } & \text { [tóláká] "abandonner (village), émigrer" } \\
& \text { [tòláká] "créer, deviner, inventer" } \\
\text { yipunu: } & \text { [ùtójà] "se fatiguer" } \\
& \text { [ùtòjà] "se battre" } \\
\text { fang (atsi): } & \text { [àbì] "cuisse d'homme" } \\
& \text { [àbí] "excrement" }
\end{array}
$$

Dans les posttextes, c'est-à-dire, la troisième et la dernière partie du Dictionnaire fang-français et français-fang, Galley (1964) passe en revue les signes et les symboles retenus pour transcrire les voyelles et les consonnes du fang. Les enseignants encourageront les élèves et les étudiants à se familiariser davantage avec ces posttextes contenant des signes et des symboles en vue de connaître avec précision les sons des langues étudiées et décrites.

\subsection{L'orthographe}

L'orthographe dont le but est de décrire le dialecte standard doit prendre en compte l'orthographe standard de l'item lexical dans lequel on trouve des variétés de forme de la langue, cf. Mdee (1990). L'enseignant connaîtra la forme 
standard et les règles orthographiques qui sont utilisées dans le dictionnaire. Il insistera sur le fait que l'orthographe revêt une importance dans tous les écrits. Et le but du dictionnaire c'est de permettre aux étudiants de vérifier l'orthographe du mot utilisé.

Pour ce qui est des règles orthographiques des langues gabonaises, nous notons, par exemple, l'harmonisation, l'assimilation des voyelles $u$ ou $i$ qui deviennent respectivement $w$ et $y$. Pour ce qui est du yipunu, par exemple, la voyelle $u$ placée devant $a$, e, et $i$ devient $w$; la voyelle $i$ devient $y$ lorsqu'elle précède les voyelles $a, e, o$ et $u$. Exemple:

$$
\begin{aligned}
& \text { muana } \rightarrow \text { mwana "enfant" } \\
& \text { uliomisa } \rightarrow \text { ulyomisa "rendre propre" } \\
& \text { mueni } \rightarrow \text { mweni "étranger" }
\end{aligned}
$$

Malgré son importance et sa valeur, l'orthographe est l'une des données les plus mal utilisées. La tâche des enseignants serait très complexe, en ce qui concerne spécialement les données orthographiques; ils devraient en être conscients. Les enseignants conseilleront aux étudiants de se référer aux pré-textes aux seules fins d'avoir des explications relatives aux données orthographiques du dictionnaire.

\subsection{La sémantique}

Dans l'évaluation critique des dictionnaires existants en langues gabonaises, le sens ne renseigne pas suffisamment sur les différents emplois du mot en contexte. Par conséquent, les usagers natifs auront des problèmes pour comprendre le contexte sémantique dans lequel les mots sont utilisés. À cet effet, le jugement de Tarp (2004: 313) est valide:

Des données sur des significations sont exclusivement fournies en vue de confirmer les usagers quel est le vrai sens du mot. Des usagers sont déjà censés avoir une idée de ce qu'ils veulent exprimer, c'est-à-dire ils connaissent déjà la signification et la nécessité juste des mots. Comme tel il est évident que le dictionnaire devrait exposer toutes les différentes significations et les sens du mot afin de satisfaire aux besoins de l'utilisateur dans n'importe quelle situation.

Puisque la complexité sémantique met en jeu plusieurs niveaux de données, le but du dictionnaire consiste à pourvoir aux élèves et aux étudiants tous les aspects sémantiques des mots, allant du sens des mots, à celui des phrases, aux relations sémantiques entre phrases dans le discours, et aux relations pragmatiques qui mettent en jeu l'utilisation des mots dans les contextes divers. L'enseignant aidera les élèves et les étudiants à vérifier si tous les sens du mot sont utilisés dans le dictionnaire. Cela nécessite au préalable qu'ils soient aptes à utiliser les procédés dictionnairiques tels que les renvois qui permettent d'éclai- 
rer le sens d'un mot et de connaître les relations sémantiques que le mot a avec les autres mots du dictionnaire. Une fois de plus, l'enseignant est appelé à encourager les élèves et les étudiants à se familiariser avec des renvois (les signes, les abréviations et les symboles) expliqués dans les pré-textes du dictionnaire.

\subsection{Le vocabulaire}

L'acquisition du vocabulaire est important pour l'apprentissage d'une langue. Les enseignants doivent aider les étudiants et les élèves à acquérir le vocabulaire par le dictionnaire. Ils les guideront dans la connaissance des propriétés ou des caractéristiques du mot. À ce propos Laufer (1992: 71) affirme que "connaître un mot implique connaître toutes ses propriétés (...). Quand une personne connaît un mot, elle connaît la prononciation, l'orthographe, les composantes morphologiques, les comportements syntaxiques dans un énoncé, les situations appropriées pour l'utiliser". Comme le dictionnaire est un livre de mots, il est l'un des moyens le plus efficace d'apprendre le vocabulaire; il permet de prendre le mot directement dans son contexte et il permet aussi aux étudiants et aux élèves de développer une connaissance générale de mots et de groupes de mots dans le but de comprendre les textes littéraires. Lorsque nous voulons apprendre, par exemple, un nouveau mot, nous consultons un dictionnaire pour connaître le mot dans son contexte. Le dictionnaire donne des exemples de phrase pour chaque mot. Pour le parler fang, les étudiants et les élèves pourraient trouver dans le Dictionnaire fang-français de français-fang de Galley (1964) plusieurs exemples de phrases accompagnant le mot. Ils pourraient avoir alors plusieurs exemples de contextes d'utilisation.

\subsection{L'étymologie}

Il a été question plus haut de préciser que la consultation du dictionnaire est une aide pour l'apprentissage du vocabulaire dans l'acquisition d'une langue, car le dictionnaire permet de connaître le mot dans son contexte. À l'instar du vocabulaire, les enseignants devraient aussi savoir attirer l'attention des étudiants et des élèves à connaître l'étymologie des mots. Puisque le dictionnaire donne la possibilité de connaître le mot: "Séparer une langue de son passé, c'est manquer la possibilité de montrer la langue dans son contexte" (Landau 2001: 132). L'étymologie permet donc d'enrichir son vocabulaire et mieux comprendre le sens des mots.

\section{Conclusion}

Dans ce travail, il a été question de voir dans quelle mesure le dictionnaire peut participer à l'enseignement des langues gabonaises. L'esprit qui a animé cette 
contribution était de présenter le contexte multilingue du Gabon, de montrer que les problèmes lexicographiques sont liés à l'éducation au Gabon: les dictionnaires en langues gabonaises sont recherchés, rarement utilisés et trouvés; le gouvernement gabonais ne subventionne même pas l'acquisition des dictionnaires existants pour stimuler les études appropriées; certains usagers ne sont pas informés de l'existence de ces outils.

Pour ce qui est du dictionnaire des langues nationales et selon le vœu exprimé par la majorité des informateurs, un outil d'enseignement comme le dictionnaire doit aller de pair avec l'intégration des langues gabonaises. Leur intégration dans le système éducatif gabonais doit se faire à partir du primaire voire dès la maternelle.

En ce qui concerne la valorisation du dictionnaire dans l'enseignement des langues gabonaises, il faut retenir que l'organisation des conférences et séminaires permettraient au public de prendre conscience de l'existence et de l'importance de cet outil dans le système éducatif.

La mise en place au Gabon des structures de recherche comme les unités lexicographiques serait un facteur déterminant pour la promotion et la sauvegarde des langues gabonaises. Ces unités lexicographiques serviraient d'interface pour la collaboration entre les lexicographes, linguistes, enseignants et apprenants (étudiants et élèves). En établissant les unités lexicographiques au Gabon, ceci aurait comme résultat la production des dictionnaires et la préservation de la diversité linguistique sous toutes ces formes.

L'utilisation de l'outil qu'est le dictionnaire serait effective chez les étudiants et les élèves en visant d'abord la formation des enseignants. Ce dernier transmettra son savoir et son expérience dictionnairique aux apprenants. Une fois que les connaissances linguistiques sont acquises chez les enseignants, on pourrait également compléter leur formation en leur enseignant plusieurs aspects de la lexicographie tels que la métalexicographie, l'histoire de la lexicographie, la structure du dictionnaire, les types de dictionnaires, groupes cibles, morphologie, phonétique, orthographe, sémantique, vocabulaire, etc.

\section{Notes}

1. Le slogan "Donne moi ton dictionnaire, j'évaluerai ta langue" (français) ou "Give me your dictionary, I will evaluate your language" (anglais) né des nombreuses discussions que j'ai eues avec le professeur Gouws lors de la 12ème conférence d'AFRILEX.

2. Le PanSALB est un projet dont le rôle est d'établir les unités lexicographiques pour les langues officielles de l'Afrique du Sud, de favoriser le multilinguisme et développer les langues qui étaient autrefois marginalisées. Grâce à ce projet l'unité lexicographique pour chacune des onze langues officielles de l'Afrique du Sud, à savoir: zoulou, xhosa, afrikaans, sepedi, anglais, tswana, sotho, tsonga, swati, venda et ndebele, a vu le jour.

3. Dans sa recherche, Joubier (2008) discute "les rapports entre la langue maternelle et la langue étrangère dans l'enseignement précoce" et affirme que dans le cas de la langue qualifiée de maternelle, l'enjeu de son acquisition est vital pour l'enfant qui grandit, se construit psychiquement et 
se construit une identité en même temps qu'il entre dans la parole et acquiert la maîtrise du langage dans ses relation sociales au contact des autres. La langue dite étrangère est par nature secondaire, sur un plan temporel d'abord, et surtout parce qu'elle apparaît comme dénuée de sens pour l'enfant, car non rattachée dans son environnement de vie à un immédiat communicatif et affectif.

4. L'inventaire linguistique géographico-administrative proposé par Kwenzi-Mikala (1998: 217) contient 62 parlers répartis en 10 groupes:

- le groupe mazuna: fan-atsi, fan-make, fang-mvaï, fan-ntumu, fan-nzaman et fan-okak;

- le groupe myene: enenga, ghalwa, mpongwe, nkomi, orungu et okoa;

- le groupe mekana-menaa: akele, ungom, lisighu, mbanwe, metombolo, seki, tumbidi, shake, wumpfu et lendambomo;

- le groupe mekona-mangote: ikota, benga, shamay, mahongwe, ndasha et bakola;

- le groupe membe (ou okande-tsogho): ghetsogo, ghepinzi, kande, ghevhovhe, ghehimbaka, ghevhiya, ebongwe et koto-a-kota;

- le groupe merye: ghisira, ghivharama, ghivhungu, yipunu, yilumbu, yisangu, ngubi, civili, yirimba et yighama;

- le groupe metye: yinzebi, yitsengi, yimvhele, yivhili, liduma, liwanzi et yibongo;

- le groupe membere: lembaama, lekanini, lindumu, lateghe et latsitseghe;

- le groupe makaana: bekwil, shiwa (ou makina) et mwese;

- le groupe baka

5. Pour l'identification des voyelles et des consonnes en langue fang (ntumu), voir. Ondo-Mébiame (1992).

\section{Références bibliographiques}

Al-Kasimi, A.M. 1977. Linguistics and Bilingual Dictionaries. Leyde: E.J. Brill.

Berri, A. et D. Pagel. 2005. La phonétique dans la classe de FLE. http://www.fdlm.org/fle/article/ 339/phonetique.php.

Bokoko, E.B. 2004. Enseignement des langues nationales au Gabon: bilan et perspectives. http://www. bibliotheque.refer.org/livre244/124423.

Brewer, C. 2006. Examining the OED [online]. http://www.english.ox.ac.uk/oed.

Bwenge, C. 1989. Lexicographical Treatment of Affixal Morphology: A Case Study of Four Swahili Dictionaries. James, G. (Éd.). 1989. Lexicographers and their Works: 5-17. Exeter: Exeter University Press.

Dolezal, F.T. et D.R. McCreary. 1999. Pedagogical Lexicography Today: A Critical Bibliography on Learners' Dictionaries with Special Emphasis on Language Learners and Dictionary Users. Lexicographica. Series Maior 96. Tübingen: Max Niemeyer.

Ekwa Ebanéga, G.-M. 2007. A Microstructural Programme for Dictionaries in Fang. Thèse de doctorat non publiée. Stellenbosch: Université de Stellenbosch.

Ekwa Ebanéga, G.-M. et F. Tomba Moussavou. 2006. Des considérations sur la lexicographie et la linguistique au Gabon. Lexikos 16: 240-250.

Ekwa Ebanéga, G.-M. et F. Tomba Moussavou. 2008. A Survey of Dictionary Use of Gabonese Students at Two South African Universities. Lexikos 18: 349-365.

Emejulu, J.D. 2001. Lexicographie multilingue et multisectorielle au Gabon: planification, stratégie et enjeux. Emejulu, J.D. (Éd.). 2001: 38-57. 
Emejulu, J.D. (Éd.). 2001. Éléments de lexicographie gabonaise. Tome I. New York: Jimacs-Hillman.

Emejulu, J.D et Y. Nzang-Bié. 1999. Linguistic Perspectives in Gabon. Communication présentée à un colloque organisé par le Summer Institute of Linguistics, Université de Dakota du Nord, Grand Forks, États-Unis, 20 juillet 1999.

Galley, P.S. 1964. Dictionnaire fang-français et français-fang, suivi d'une grammaire fang. Neuchâtel: Henri Messeiller.

Gates, E. 1997. A Survey of the Teaching of Lexicography: 1979-1995. Dictionaries 18: 66-93.

Gouws, R.H. 2001. Formation lexicographique: approches et thèmes. Emejulu, J.D. (Éd.). 2001: 95134.

Hadebe, S. 2004. Improving Dictionary Skills in Ndebele. Lexikos 14: 89-104.

Hausmann, F.J. 1986. The Training and Professional Development of Lexicographers in Germany. Ilson, R.E. (Éd.). 1986. Lexicography. An Emerging International Profession: 101-110. Manchester: Manchester University Press.

Householder, F.W. 1962. Summary Report. Householder, F.W. et S. Saporta (Éds.). 1962. Problems in Lexicography: 279-282. Bloomington: Indiana University/Le Havre: Mouton.

Joubier, A. 2008. Les rapports entre langue maternelle et langue étrangère. http://www.edufle.net/Lesrapports-entre-la-langue.

Kipfer, B.A. 1987. Dictionaries and the Intermediate Student: Communicative Needs and the Development of User Reference Skills. Cowie, A.P. (Éd.). 1987. The Dictionary and the Language Learner. Papers from the EURALEX Seminar at the University of Leeds, 1-3 April 1985: 4454. Lexicographica. Series Maior 17. Tübingen: Max Niemeyer.

Kwenzi-Mikala, J.T. 1998. Parlers du Gabon: Classification du 11.12.97. Raponda-Walker, A. (Éd.). 1998. Les langues du Gabon: 217. Classiques africains. Libreville: Éditions Raponda-Walker.

Landau, S.I. 2001. Dictionaries. The Art and Craft of Lexicography. New York: Cambridge University Press.

Laufer, B. 1992. Corpus-Based versus Lexicographer Examples in Comprehension and Production of New Words. Tommola, H. et al. (Éds.). 1992. EURALEX '92 Proceedings I-II: Papers Submitted to the 5th EURALEX International Congress on Lexicography in Tampere, Finland: 71-76. Tampere: Université de Tampere.

Mabika Mbokou, L. 2001. Le rôle du dictionnaire dans le système éducatif. Emejulu, J.D. (Éd.). 2001: 206-222.

Malmberg, B. 1954. La phonétique. Paris: Presses Universitaires de France.

McArthur, T. 1986. Worlds of Reference. Lexicography, Learning and Language from the Clay Tablets to the Computer. Cambridge: Cambridge University Press.

McKean, E. 2000. Dictionary Activities in the Elementary Classroom: News for Lexicographers. Dictionaries 21: 81-89.

Mdee, J.S. 1990. Dictionaries and the Standardization of Spelling in Swahili. Lexikos 9: 119-134.

Mihindou, G.-R. 2001. Apports des missionnaires à la lexicographie gabonaise: Dictionnaires bilingues fang-français/français-fang; français-yipounou/yipounou-français; français-mpongwé. Emejulu, J.D. (Éd.). 2001: 7-37.

Moyo, T. 2002. Mother Tongues versus an Ex-colonial Language as Media of Instruction and the Promotion of Multilingualism: The South African Experience. South African Journal of African Languages 22(2): 149-160. 
Ndinga-Koumba-Binza, H.S. 2005. Politique linguistique et éducation au Gabon: un état des lieux. Journal of Education 4(1): 65-78. Réduit: Mauritius Institute of Education.

Nyangone Assam, B. 2001. La lexicographie dans l'apprentissage des langues gabonaises. Emejulu, J.D. (Éd.). 2001: 187-205.

Nyangone Assam, B. et P.A. Mavoungou. 2000. Lexicography in Gabon: A Survey. Lexikos 10: 252274.

Nzang-Bié, Y. 2004. La problématique de la révision des dictionnaires anciens: le cas du Dictionnaire fang-français et français-fang de Samuel Galley (1964). Communication non publiée. Libreville: GRELACO, Université Omar Bongo.

Ondo-Mébiame, P. 1992. De la phonologie à la morphologie du fang-ntumu parlé à Aboumézok (bantu A.78). 2 volumes. Thèse de doctorat (NR). Bruxelles: Université Libre de Bruxelles.

Tarp, S. 2004. Reflections on Dictionaries Designed to Assist Users with Text Production in a Foreign Language. Lexikos 14: 299-325.

Tomba Moussavou, F. 2007. Metalexicographic Criteria for a Monolingual Descriptive Dictionary Presenting the Standard Variety of Yipunu. Thèse de doctorat non publiée. Stellenbosch: Université de Stellenbosch.

Wiegand, H.E. 1984. On the Structure and Contents of a General Theory of Lexicography. Hartmann, R.R.K (Éd.). 1984. LEXeter '83 Proceedings. Papers from the International Conference on Lexicography at Exeter, 9-12 September 1983: 13-30. Lexicographica. Series Maior 1. Tübingen: Max Niemeyer. 